\title{
GENERALIZED PERMUTATION PATTERNS - A SHORT SURVEY
}

\author{
EINAR STEINGRÍMSSON
}

\begin{abstract}
An occurrence of a classical pattern $p$ in a permutation $\pi$ is a subsequence of $\pi$ whose letters are in the same relative order (of size) as those in $p$. In an occurrence of a generalized pattern, some letters of that subsequence may be required to be adjacent in the permutation. Subsets of permutations characterized by the avoidance - or the prescribed number of occurrencesof generalized patterns exhibit connections to an enormous variety of other combinatorial structures, some of them apparently deep. We give a short overview of the state of the art for generalized patterns.

Key words: Permutation, pattern, Generalized permutation patterns, pat-
\end{abstract} tern avoidance.

\section{Introduction}

Patterns in permutations have been studied sporadically, often implicitly, for over a century, but in the last two decades this area has grown explosively, with several hundred published papers. As seems to be the case with most things in enumerative combinatorics, some instances of permutation patterns can be found already in MacMahon's classical book from 1915, Combinatory Analysis [48]. In the seminal paper Restricted permutations of Simion and Schmidt [56] from 1985 the systematic study of permutation patterns was launched, and it now seems clear that this field will continue growing for a long time to come, due to its plethora of problems that range from the easy to the seemingly impossible, with a rich middle ground of challenging but solvable problems. Most important, perhaps, for the future growth of the subject, is the wealth of connections to other branches of combinatorics, other fields of mathematics, and to other disciplines such as computer science and physics.

Whereas an occurrence of a classical pattern $p$ in a permutation $\pi$ is simply a subsequence of $\pi$ whose letters are in the same relative order (of size) as those in $p$, in an occurrence of a generalized pattern, some letters of that subsequence may be required to be adjacent in the permutation. For example, the classical pattern 1-2-3-4 simply corresponds to an increasing subsequence of length four, whereas an occurrence of the generalized pattern 1-23-4 would require the middle two letters of that sequence to be adjacent in $\pi$, due to the absence of a dash between 2 and 3. Thus, the permutation 23145 contains 1-2-3-4 but not 1-23-4. Note that for the classical patterns, our notation differs from the usual one, since the dashes we have between every pair of adjacent letters in a classical pattern are usually omitted when only classical patterns are being considered.

It is well known that the number of permutations of length $n$ avoiding any one classical pattern of length 3 is the $n$-th Catalan number, which counts a myriad different combinatorial objects. There are many other results in this direction, relating pattern avoiding permutations to various other combinatorial structures, either via bijections, or by such classes of permutations being equinumerous to the structures

The work presented here was supported by grant no. 060005012/3 from the Icelandic Research Fund. 
in question without there being a known bijection. Counting permutations according to the number of occurrences of generalized patterns one comes up with a vast array of known sequences, such as the Euler numbers, Stirling numbers of both kinds, Motzkin numbers, Entringer numbers, Schröder numbers, Fibonacci numbers, Pell numbers and many more. Also, one often finds lesser known sequences that are nevertheless related to known structures, such as directed animals, planar maps, permutation tableaux, various kinds of trees and involutions in $S_{n}$, to name a few. Thus, generalized patterns provide a significant addition to the already sizable flora of classical patterns and their connections to other combinatorial structures.

In fact, due to their great diversity, the non-classical generalized patterns are likely to provide richer connections to other combinatorial structures than the classical ones do. Supporting this is the fact that the recently proved Stanley-Wilf conjecture - which gives a strong bound for the growth rate of the number of permutations of length $n$ avoiding a classical pattern - does not hold for some generalized patterns.

This paper is organized as follows: In Section 2 we introduce definitions and in Section 3 we mention implicit appearances of generalized patterns in the literature. In Sections 4 and 5 we survey what is known about the avoidance of generalized patterns of length three and four, respectively. In Section 6 we give some examples where generalized patterns have shown up in very different contexts, establishing connections to various other combinatorial structures, some of which seem quite deep. Section 7 lists several instances of so-called barred patterns that turn out to be equivalent to generalized patterns and Section 8 deals with asymptotics for avoidance of generalized patterns. Finally, in Section 9, we mention some further generalizations of the generalized patterns.

\section{Some definitions}

If a permutation $\pi=a_{1} a_{2} \ldots a_{n}$ contains the pattern 1-23 then clearly the reverse of $\pi$, that is $a_{n} a_{n-1} \ldots a_{1}$, contains the reverse of $1-23$, which is the pattern $32-1$. Since taking the reverse of a permutation is a bijection on the set of permutations of length $n$, the number of permutations avoiding a pattern $p$ equals the number of permutations avoiding the reverse of $p$. More generally, the distribution - on the set of permutations of length $n$ - of the number of occurrences of a pattern $p$ equals the distribution for the reverse of $p$. The same is true of the bijection sending a permutation $\pi=a_{1} a_{2} \ldots a_{n}$ to its complement $\pi^{c}=b_{1} b_{2} \ldots b_{n}$, where $b_{i}=n+1-a_{i}$. (When we take the complement of a pattern we leave the dashes in place, so the complement of 1-342 is 4-213.) These two transformations, together with their compositions, generate a group of order 4 on the set of patterns, and we say that two patterns belong to the same symmetry class if one is transformed into the other by an element of this group. As an example, the patterns 2-31, 2-13, 13-2 and 31-2 form an entire symmetry class. ${ }^{1}$

Clearly, two patterns in the same symmetry class have the same properties with respect to the number of permutations avoiding them, and more generally when it comes to the number of permutations with $k$ occurrences of a pattern, for any $k$. However, it does happen that two patterns not belonging to the same symmetry class have the same avoidance. Thus, two patterns are said to belong to the same Wilf class if they have the same avoidance, that is, if the number of permutations of length $n$ that avoid one is the same as that number for the other. Clearly, a Wilf class is a union of symmetry classes (if we define both as equivalence classes).

\footnotetext{
${ }^{1}$ In the case of classical patterns, taking the inverse of a pattern is well defined and preserves avoidance, so patterns that are each other's inverses belong to the same symmetry class. This is not the case for generalized patterns.
} 
For example, although the classical patterns of length 3 belong to two symmetry classes (represented by 1-2-3 and 1-3-2, respectively), they all belong to the same Wilf class, since their avoidance is given by the Catalan numbers. For classical patterns, much is known about Wilf classes for patterns of length up to 7 , but a general solution seems distant. For the generalized patterns, much less is known. The best reference to date is probably [18]. As an example, the patterns 1-23 and 1-32 belong to the same Wilf class (the avoidance counted by the Bell numbers in both cases) but not the same symmetry class.

\section{Generalized patterns in the literature}

Generalized patterns have shown up implicitly in the literature in various places, and subsets of these have been studied in some generality. Namely, Simion and Stanton [57] essentially studied the patterns 2-31, 2-13, 31-2, and 13-2 and their relation to a set of orthogonal polynomials generalizing the Laguerre polynomials, and one of these patterns also played a crucial role in the proof by Foata and Zeilberger [31] that Denert's statistic is Mahonian. A permutation statistic is Mahonian if it has the same distribution - on the set of permutations of length $n$ for each $n$-as the number of inversions.

Goulden and Jackson give an exponential generating function (EGF) for the number of permutations avoiding the pattern 123 (no dashes), in the book Combinatorial Enumeration [36, Exercise 5.2.17a, p. 310]. The formula is

$$
\left(\sum_{n \geq 0} \frac{x^{3 n}}{(3 n) !}-\sum_{n \geq 0} \frac{x^{3 n+1}}{(3 n+1) !}\right)^{-1}
$$

Although this does not seem to be mentioned in [36], the obvious generalization holds. Namely, the EGF for the number of permutations avoiding the dashless pattern $12 \cdots k$ is obtained by replacing 3 by $k$ in (1). It is pointed out in [45, Section 3] that this general result can be obtained through an inclusion/exclusion argument similar to one given in [45].

The dashless patterns of length 3 also appeared earlier, implicitly, as the valleys (213 and 312), the peaks (132 and 231), the double ascents 123 and the double descents 321 in a permutation, the study of which was pioneered by Françon and Viennot [33], and which is intimately related to Flajolet's [29] generation of Motzkin paths by means of certain continued fractions. This will be mentioned later, in Section 6 , in connection with related, recent developments.

Also, the alternating permutations, which have been studied for a long time $[2,3]$, are permutations that avoid the patterns 123 and 321, (with the additional restriction that the first two letters of the permutation be in decreasing order; otherwise they are reverse alternating. In fact, this extra restriction is equivalent to the avoidance of the pattern [12) as defined in [4]).

Generalized patterns were first defined explicitly, in full generality, in the paper Generalized permutation patterns and a classification of the Mahonian statistics [4], where it was shown that almost all Mahonian permutation statistics in the literature at that time (up to a certain bijective correspondence translating "excedance based" statistics to "descent based" statistics; see [4, 20]) could be written as linear combinations of generalized patterns. All but one of these statistics could be expressed as combinations of patterns of length at most 3 . The odd one out was a statistic defined by Haglund [37], which, after translation by the bijection mentioned above, could be expressed as a combination of patterns of length 4 or less (see [4]). Although all the possible Mahonian statistics based on generalized patterns of length at most 3 were listed in [4], proofs were not given that all of them 
were indeed Mahonian. These proofs were later supplied by Foata and Zeilberger [32], who solved some of the conjectures with bijections, but others using the "Umbral Transfer Matrix Method" of Zeilberger [63]. The remaining conjectures in [4], concerning a slight generalization of generalized patterns, were proved bijectively by Foata and Randrianarivony [30].

\section{Avoidance (and occurrences) of generalized patterns of length 3}

The study of avoidance of generalized patterns - along the lines of the work of Simion and Schmidt [56] for the classical patterns - was initiated by Claesson in the paper Generalized pattern avoidance [16], where the enumeration was done for avoidance of any single pattern of length 3 with exactly one dash. These patterns fall into three equivalence classes with respect to avoidance. One of these classes, consisting of the patterns 2-31, 2-13, 31-2 and 13-2, has the same avoidance as both of the classes of classical patterns of length 3 , and thus has avoidance enumerated by the Catalan numbers. In fact, avoiding 2-31 is equivalent to avoiding 2-3-1, as shown in [16], and likewise for the other three patterns. This is obviously true also for the patterns 21 and 2-1, since the only permutation with no descent and no inversion is the increasing permutation $123 \ldots n$. It is shown by Hardarson [38] that this can not occur for patterns of length greater than three, that is, two different patterns of length more than three cannot be avoided by the same permutations. The other two classes of one-dash patterns of length three, consisting of the patterns 1-23 and 1-32 and their respective sets of equivalent patterns, are enumerated by the Bell numbers, counting partitions of sets. This was proved bijectively in each case in [16].

The fact that the Bell numbers count permutations avoiding 1-23 (and some other generalized patterns) has interesting implications. Namely, this shows that the Stanley-Wilf conjecture (proved by Marcus and Tardos [49] in 2004) does not hold for some generalized patterns. This ex-conjecture says that for any classical pattern $p$ the number of permutations of length $n$ avoiding $p$ is bounded by $C^{n}$ for some constant $C$. This is easily seen to fail for the Bell numbers, whose exponential generating function is $e^{\left(e^{x}-1\right)}$. Apart from this, nothing seems to be known about growth rates of the number of permutations avoiding generalized patterns.

That the Bell numbers count the avoidance of 1-23 also implies the falseness for generalized patterns of another conjecture, by Noonan and Zeilberger [52] (but first mentioned by Gessel [34]), that the number of permutations avoiding a classical pattern is polynomially recursive, that is, satisfies a recursion

$$
P_{0}(n) f(n)=P_{1}(n) f(n-1)+P_{2}(n) f(n-2)+\cdots+P_{k}(n) f(n-k)
$$

where $k$ is a constant and each $P_{i}$ is a polynomial. This conjecture, however, is largely believed to be false even for classical patterns, although a counterexample is still missing.

Claesson [16] also enumerated the avoidance of three classes of pairs of generalized patterns of length 3 with one dash each. These turned out to be equinumerous with non-overlapping set partitions (counted by the Bessel numbers), involutions and Motzkin paths, respectively. Enumerative equivalences among the class of patterns corresponding to non-overlapping partitions, together with the connection to set partitions, naturally led to the definition of monotone partitions in [16].

In [18], Claesson and Mansour then completed the enumeration of permutations avoiding any pair of generalized patterns with one dash each. They also conjectured enumerative results for avoidance of any set of three or more such patterns. These conjectures were proved for sets of size three by Bernini, Ferrari and Pinzani [5], 
and by Bernini and Pergola [7] for the sizes 4, 5 and 6, the remaining sizes being rather trivial.

Elizalde and Noy [28] treated the dashless patterns (which they call "consecutive patterns"), that is, patterns with no dashes, and gave generating functions enumerating permutations according to the number $k$ of occurrences of a pattern. This is a much stronger result than enumerating permutations avoiding a pattern, which is just the special case $k=0$. In particular, they enumerated the avoidance of both Wilf classes of dashless patterns of length 3 , and gave differential equations satisfied by the generating functions for three of the seven Wilf classes of patterns of length 4 (see the next section). As mentioned before, their result in the special case of avoidance of the pattern 123, was obtained already in the book Combinatorial Enumeration by Goulden and Jackson [36, Exercise 5.2.17a].

For subsets of two or more dashless patterns of length 3, Kitaev [41] and Kitaev and Mansour [46, 47] gave direct formulas for almost every case, and recursive formulas for the few remaining ones. Examples of the formulas thus obtained are $C_{k}+C_{k+1}$ where $C_{k}$ is the $k$-th Catalan number, the central binomial coefficients $\left(\begin{array}{c}2 n \\ n\end{array}\right)$, and the Entringer numbers, which also count certain permutations starting with a decreasing sequence and then alternating between ascents and descents.

Thus, the avoidance of any set of generalized patterns of length 3 has been understood, in most cases in the sense of explicit formulas or generating functions, or at least in terms of recursively defined functions.

In [17], Claesson and Mansour found the number of permutations with exactly one, two and three occurrences, respectively, of the pattern 2-31. They used the connection between continued fractions and Motzkin paths (see Section 6) that had been used in [20] to give a continued fraction for a generating function counting occurrences of 2-31, among other things. Later, Parviainen [54] showed how to use bicolored Motzkin paths to give a continued fraction counting permutations of length $n$ according to the number of occurrences of 2-31. He gave an algorithm for finding an explicit formula, and gave this explicit formula (always a rational function in $n$ times a binomial coefficient of the form $\left(\begin{array}{c}2 n \\ n-a\end{array}\right)$ ) for each $n \leq 8$. Finally, Corteel and Nadeau [22] found a bijective proof of the fact, first proved in [17], that the number of permutations of length $n$ with exactly one occurrence of 2-31 is $\left(\begin{array}{c}2 n \\ n-3\end{array}\right)$. They exploited the connection between generalized patterns and permutation tableaux. For more about that connection, see Section 6 .

\section{Patterns of length 4}

The classical patterns of length 4 fall into three Wilf classes, represented by the patterns 1-2-3-4, 1-3-4-2 and 1-3-2-4. The avoidance of the first two has been solved (the first by Gessel [34], the second by Bóna [8]), but 1-3-2-4 still remains to be understood, although some noteworthy progress was recently made by Albert et al. in [1].

For generalized patterns of length 4 (other than the classical ones), the situation is much more complicated than for those of length 3 , as is to be expected. There are 48 symmetry classes, and computer experiments show that there are at least 24 Wilf classes, but their exact number does not seem to have been determined yet.

As mentioned above, for the dashless patterns of length 4, Elizalde and Noy [28] gave differential equations satisfied by the generating functions for the number of occurrences of three out of seven Wilf classes, namely the classes containing 1234, 1243 and 1342, respectively. The remaining classes are represented by 2413, 2143, 1324 and 1423. Note that a Wilf class is a class of patterns with the same avoidance, whereas Elizalde and Noy proved that in each Wilf class of dashless patterns of length four, all patterns have the same distribution. That is, the number 
of permutations of length $n$ with $k$ occurrences of a pattern is the same for two patterns in the same Wilf class in this case, but not in general.

Kitaev [45, Theorem 13] found an expression for the exponential generating function (EGF) for the avoidance of $\sigma-k$, where $\sigma$ is any dashless pattern and $k$ is larger than all the letters in $\sigma$, in terms of the EGFs for avoidance of the pattern $\sigma$. In particular, if $\sigma$ is any dashless pattern of length 3, this, together with the results of Elizalde and Noy [28], yields explicit formulas for the EGFs for the avoidance of $\sigma-4$, where $\sigma$ is any dashless pattern of length 3 (although one of these formulas involves the integral $\int_{0}^{x} e^{-t^{2}} d t$ ). Since there are precisely two Wilf classes for dashless patterns of length 3, this gives the avoidance of two Wilf classes of patterns of length 4 .

Also, Callan [14] has given two recursive formulas for the number of permutations avoiding 31-4-2.

These seem to be all the enumerative results so far for patterns of length 4 . For the non-classical patterns we thus have formulas for the avoidance of six Wilf classes out of (at least) 24, whereas for the classical patterns of length 4 there are formulas for two out of three classes. However, there is no reason to assume that the non-classical patterns should be harder to deal with than the classical ones, so explicit results for the avoidance of more such patterns should not be considered out of reach.

\section{Generalized patterns appearing in other contexts}

In Section 1 we mentioned the connection between dashless patterns as valleys, peaks, etc., in permutations, and Flajolet's [29] generation of Motzkin paths by means of continued fractions. Using results from Flajolet's paper [29], Clarke, Steingrímsson and Zeng [20, Corollary 11] found a continued fraction capturing, among other things, the distribution of permutations according to the number of occurrences of 2-31. This was made explicit in [17, Corollary 23]. A polynomial formula for the joint distribution of descents and 2-31 was conjectured by Steingrímsson and Williams (unpublished), after Williams [61, Corollary 5.3] had shown that formula to count permutations according to weak excedances and alignments which Williams was studying in connection with so called permutation tableaux (for definitions see $[58,61]$ ). This conjecture (part of a much larger conjecture later proved in [58]) was first proved by Corteel [21]. The formula is as follows (see Corollary 30 in [58]):

The number of permutations of length $n$ with $k-1$ descents and $m$ occurrences of the pattern 2-31 is equal to the coefficient of $q^{m}$ in

$$
q^{-k^{2}} \sum_{i=0}^{k-1}(-1)^{i}[k-i]^{n} q^{k i}\left(\left(\begin{array}{c}
n \\
i
\end{array}\right) q^{k-i}+\left(\begin{array}{c}
n \\
i-1
\end{array}\right)\right) .
$$

Here, $[k-i]$ is the $q$-bracket defined by $[m]=\left(1+q+\cdots+q^{m-1}\right)$. This is the only known polynomial formula for the entire distribution of a pattern of length greater than 2. The two cases of length 2 correspond to the Eulerian numbers, counting descents, and the coefficients of the $q$-factorial

$$
[n] !=(1+q)\left(1+q+q^{2}\right) \cdots\left(1+q+q^{2}+\cdots+q^{n-1}\right),
$$

which count permutations according to the number of inversions. A descent is an occurrence of the pattern 21 and an inversion is an occurrence of 2-1.

Moreover, this connection between patterns and permutation tableaux also led to the discovery, by Corteel [21] (see also [23, 24]), of a connection between the permutation tableaux and the partially asymmetric exclusion process (PASEP), an 
important model in statistical mechanics. In particular, the distribution of permutations of length $n$ according to number of descents and number of occurrences of the pattern 2-31 equals a probability distribution studied for the PASEP.

In [19], a bijection is given between the permutations of length $n$ avoiding both $2-41-3$ and $3-1-4-2$ on one hand, and so called $\beta(1,0)$-trees on the other. The $\beta(1,0)$-trees are rooted plane trees with certain labels on their vertices. These trees were defined by Jacquard and Schaeffer [40], who described a bijection from rooted nonseparable planar maps to a set of labeled plane trees including the $\beta(1,0)$-trees. These trees represent, in a rather transparent way, the recursive structure found by Brown and Tutte [10] on planar maps. As it turns out, the bijection given in [19] simultaneously translates seven different statistics on the permutations to corresponding statistics on the $\beta(1,0)$-trees. In fact, the permutations avoiding $2-41-3$ and 3-1-4-2 seem to be more closely related structurally to the $\beta(1,0)$-trees - and thus to the planar maps involved - than the two-stack sortable permutations that had previously been shown to be in bijection with the planar maps in question (see Section 7). Earlier, Dulucq, Gire and West [25] constructed a generating tree for the permutations avoiding 2-4-1-3 and 3-14-2 that they showed to be isomorphic to a generating tree for rooted nonseparable planar maps. (Clearly, permutations avoiding these two patterns are equinumerous with the permutations avoiding 3-1-4-2 and 2-41-3, treated in [19].) However, instead of the pattern 3-14-2 they used a so-called barred pattern, which we treat in Section 7, and they only showed their bijection, which is different from the one in [19], to preserve two different statistics, rather than the seven statistics in [19].

\section{Generalized patterns in disguise}

As mentioned above, generalized patterns have occurred implicitly in several places in the literature, even before the systematic study of classical permutation patterns. However, they have also appeared as so-called barred patterns, which sometimes, but not always, turn out to be equivalent (in terms of avoidance) to some generalized patterns. An example of a barred pattern is $4-\overline{2}-1-3$. A permutation $\pi$ is said to avoid this pattern if it avoids the pattern 3-1-2 (corresponding to the unbarred elements 4-1-3) except where that pattern is part of the pattern 4-2-1-3.

Gire [35] showed that the Baxter permutations, originally defined in a very different way [15], are those avoiding the two barred patterns $4-1-\overline{3}-5-2$ and $2-5-\overline{3}-1-4$. It is easy to show that avoiding $4-1-\overline{3}-5-2$ is equivalent to avoiding $3-14-2$ and avoiding $2-5-\overline{3}-1-4$ is equivalent to avoiding $2-41-3$. Thus, the Baxter permutations are precisely those that avoid both 3-14-2 and 2-41-3. In fact, this was pointed out in Erik Ouchterlony's thesis [53, p. 5].

The barred pattern $4-1-\overline{3}-5-2$ also shows up in [25], where Dulucq, Gire and West treated so called nonseparable permutations (bijectively related to rooted nonseparable planar maps), which they characterized by the avoidance of 2-4-1-3 and $4-1-\overline{3}-5-2$, the latter one being equivalent to $3-14-2$ as mentioned in the previous section.

Also, in [9], Bousquet-Mélou and Butler deal with the pattern 2-1- $\overline{3}-5-4$, avoiding which is easily shown to be equivalent to avoiding 2-14-3. Permutations avoiding that pattern and 1-3-2-4 are called forest-like permutations in [9]. It is also mentioned there that avoiding $2-1-\overline{3}-5-4$ (and thus $2-14-3$ ) is equivalent to avoiding 2-1-4-3 with Bruhat condition $(1 \leftrightarrow 4)$ in the terminology of Woo and Yong, who conjectured [62] that a Schubert variety is locally factorial if and only if its associated permutation avoids these two patterns. That conjecture was proved in $[9]$. 
Not all barred patterns can be expressed in terms of generalized patterns, however. For example, West [59] showed that two-stack sortable permutations are characterized by the simultaneous avoidance of 2-3-4-1 and the barred pattern $2-\overline{5}-3-4-1$, and it is easy to show that no generalized pattern is avoided by the same permutations as those avoiding $2-\overline{5}-3-4-1$. It is also easy to show that there is no pair of generalized patterns of length 4 that is avoided by the same permutations as those that avoid $2-3-4-1$ and $2-\overline{5}-3-4-1$. A consequence of this is that two-stack sortability of a permutation cannot be characterized by the avoidance of a set of generalized patterns. This is because there are precisely two permutations of length four that are not two-stack sortable, namely 2341 and 3241, so such a set would have to contain two generalized patterns whose underlying permutations (obtained by disregarding the dashes in the patterns) were 2341 and 3241 . It is easy to check, by computer, that no such pair will do the job.

Also Callan [13] has shown that the number of permutations avoiding 31-4-2 is the same as the number avoiding the barred pattern $3-\overline{5}-2-4-1$, although these are not the same permutations. (As mentioned above, Callan [14] has given two recursive formulas for this number.)

An obvious open problem here is to determine when avoiding a barred pattern is equivalent to avoiding a generalized pattern.

\section{Asymptotics}

As mentioned before, the Stanley-Wilf conjecture, proved by Marcus and Tardos [49], says that the number of permutations of length $n$ avoiding any given classical pattern $p$ is bounded by $C^{n}$ for some constant $C$ depending only on $p$.

In [27], Elizalde studies asymptotics for the number of permutations avoiding some generalized patterns and concludes that, in contrast to the classical patterns, there probably is "a big range of possible asymptotic behaviors." Although much work remains to be done here, and although it is not clear how varied this behavior can be, the extremes are already known. Namely, whereas the number of permutations of length $n$ avoiding a classical pattern is bounded by $c^{n}$ for a constant $c$, Elizalde [27, Theorem 4.1] shows that the number $\alpha_{n}(\sigma)$ of permutations of length $n$ avoiding a dashless (or consecutive) pattern $\sigma$ of length at least three satisfies

$$
c^{n} n !<\alpha_{n}(\sigma)<d^{n} n !
$$

for some constants $c$ and $d$ (where, clearly, $0<c, d<1$ ).

An interesting open question related to this is which generalized patterns, apart from the classical ones, satisfy the Stanley-Wilf conjecture. It has been pointed out by Hardarson [39] that a pattern containing a block with at least two letters $a$ and $b$, with $a<b$, and a letter $x$ in some other block, with $x<a$ or $b<x$, can not satisfy the Stanley-Wilf conjecture, because there will be at least as many permutations avoiding it as there are permutations that avoid 1-23, and the number of such permutations is known not to satisfy the Stanley-Wilf conjecture (these are the Bell numbers). Thus, essentially the only open cases left are the patterns 2-3-41, $3-2-41,3-41-2$ and 2-41-3, which all have the same avoidance for $n \leq 10$. If these patterns turn out not to satisfy the Stanley-Wilf conjecture, that would prove the tempting conjecture that a generalized pattern satisfies the Stanley-Wilf conjecture if and only if it is avoided by the same permutations as the classical pattern with the same underlying permutation. As mentioned in Section 4, it has been shown by Hardarson [38] that this happens only for patterns of length 3 or less. 


\section{Further generalizations}

Kitaev, in [45], introduced a further generalization of generalized patterns (GPs), namely the partially ordered generalized patterns or POGPs. These are GPs where some letters may be incomparable in size. An example of such a pattern is 3-12-3, an occurrence of which consists of four letters, the middle two adjacent (and in increasing order) and the first and the last both greater than the middle two, with no condition on the relative sizes of the first and the last letter. Avoiding the pattern $3-12-3$ is equivalent to avoiding both 3-12-4 and 4-12-3. Indeed, an occurrence of 3-12-3 is equivalent to an occurerence of either $3-12-4$ or $4-12-3$. In general, a POGP is equal, as a function counting occurrences, to a sum of GPs.

In [38], Hardarson finds EGFs for the avoidance of $k-\sigma-k$, where $\sigma$ is any dashless partially ordered pattern and $k$ is larger than any letter in $\sigma$, in terms of the EGF for the avoidance of $\sigma$. In the special case where $\sigma=12$ he gives a bijection between permutations avoiding 3-12-3 and bicolored set partitions, that is, all partitions of a set where each block in the partition has one of two possible colors. Also, for $\sigma=121$, he gives a bijection between permutations of length $n+1$ avoiding 3-121-3 and the Dowling lattice on $\{1,2, \ldots, n\}$.

One interesting, and curious, result arising from Kitaev's study of POGPs in [45] is that knowing the EGF for the avoidance of a dashless pattern $p$ is enough to find the EGF for the entire distribution of the maximum number of non-overlapping occurrences of $p$. Two occurrences of $p$ in a permutation $\pi$ are non-overlapping if they have no letter of $\pi$ in common. For example, the permutation 4321 has three descents (occurrences of 21), but only two non-overlapping descents. Namely, Kitaev [45] proves the following theorem:

Theorem (Kitaev [45], Theorem 32). Let $p$ be a dashless pattern. Let $A(x)$ be the $E G F$ for the number of permutations that avoid $p$ and let $N(\pi)$ be the maximum number of non-overlapping occurrences of $p$ in $\pi$. Then

$$
\sum_{\pi} y^{N(\pi)} \frac{x^{|\pi|}}{|\pi| !}=\frac{A(x)}{1-y((x-1) A(x)+1)},
$$

where the sum is over all permutations of lengths $0,1,2, \ldots$

Alternative proofs, and some extensions, of Theorem 9 were given by Mendes [50] and by Mendes and Remmel [51], using the theory of symmetric functions.

A generalization in a different direction is the study of generalized patterns on words, that is, on permutations of multisets. Research in this area has only recently taken off, even in the case of classical patterns. For the generalized patterns, see $[6,12,42]$.

Finally, Burstein and Lankham have considered barred generalized patterns, in relation to patience sorting problems (see [11], which also has further references).

\section{References}

[1] M.H. Albert, M. Elder, P. Westcott and M. Zabrocki: On the Stanley-Wilf limit of 4231avoiding permutations and a conjecture of Arratia, Adv. Appl. Math. 36(2) (2006), 96-105.

[2] D. André: Développements de sec x et tan x, Comptes Rendus Acad. Sci., Paris 88 (1879), 965-967.

[3] d. André :Mémoire sur les permutations alternées.” J. Math. 7 (1881), 167-184.

[4] E. Babson, E. Steingrímsson: Generalized permutation patterns and a classification of the Mahonian statistics, Sém. Lothar. Combin., B44b (2000), 18 pp.

[5] A. Bernini, L. Ferrari and R. Pinzani: Enumerating Permutations Avoiding Three BabsonSteingrímsson Patterns, Annals Combin. 9 (2005), 137-162.

[6] A. Bernini, L. Ferrari, R. Pinzani: Enumeration of some classes of words avoiding two generalized patterns of length three, preprint, arXiv:0711.3387v1. 
[7] A. Bernini, E. Pergola: Enumerating permutations avoiding more than three BabsonSteingrímsson patterns, J. Integer Seq. 10 (2007), Article 07.6.4, 21 pp. (electronic).

[8] Miklós Bóna: Exact enumeration of 1342-avoiding permutations; A close link with labeled trees and planar maps, J. Combin. Theory Ser. A 80 (1997), 257-272.

[9] M. Bousquet-Mélou and S. Butler:-like permutations, Ann. Comb. 11 (2007) 335-354.

[10] W. G. Brown and W. T. Tutte: On the enumeration of rooted non-separable planar maps, Canad. J. Math. 16 (1964), 572-577.

[11] A. Burstein and I. Lankham: Restricted Patience Sorting and Barred Pattern Avoidance, preprint, arXiv:math.CO/0512122.

[12] A. Burstein, T. Mansour: Words restricted by 3-letter generalized multipermutation patterns, Ann. Comb. 7 (2003) 1-14.

[13] D. Callan: A Wilf equivalence related to two stack sortable permutations, preprint, arXiv:math/0510211v1.

[14] D. Callan: A Combinatorial Interpretation of the Eigensequence for Composition, J. Integer Sequences 9 (2006), Article 06.1.4.

[15] F.R.K. Chung, R.L. Graham, V.E. Hoggatt, Jr. and M. Kleiman: The number of Baxter permutations, J. Combin. Theory, Ser. A 24 (1978), 382-394.

[16] A. Claesson: Generalized Pattern Avoidance, Europ. J. Combin. 22 (2001), 961-971.

[17] A. Claesson and T. Mansour: Counting Occurrences of a Pattern of Type $(1,2)$ or $(2,1)$ in Permutations, Adv. Appl. Math. 29 (2002), 293-310.

[18] A. Claesson and T. Mansour: Enumerating permutations avoiding a pair of BabsonSteingrímsson patterns, Ars Combinatoria 77 (2005), 17-31.

[19] A. Claesson, S. Kitaev and E. Steingrímsson: Two-stack sortable permutations, $\beta(1,0)$-trees, planar maps and generalized permutation patterns, in preparation.

[20] R. J. Clarke, E. Steingrímsson and J. Zeng: New Euler-Mahonian statistics on permutations and words, Adv. in Appl. Math. 18 (1997), 237-270.

[21] S. Corteel, Crossings and alignements of permutations, Adv. Appl. Math. 38 (2007), 149-163.

[22] S. Corteel and P. Nadeau: Bijections for permutation tableaux, Europ. J. Combin., to appear.

[23] S. Corteel and L.K. Williams: Tableaux combinatorics for the asymmetric exclusion process, Adv. Appl. Math. 39 (2007), 293-310.

[24] S. Corteel and L.K. Williams: A Markov chain on permutations which projects to the PASEP, Internat. Math. Research Notices 2007, article ID mm055.

[25] S. Dulucq, S. Gire, and J. West: Permutations with forbidden subsequences and nonseparable planar maps, Disc. Math. 153(1-3) (1996), 85-103.

[26] S. Dulucq and O. Guibert: Stack words, standard tableaux and Baxter permutations, Disc. Math. 157 (1996), 91-106.

[27] S. Elizalde: Asymptotic enumeration of permutations avoiding generalized patterns, Adv. in Appl. Math. 36 (2006), 138-155.

[28] S. Elizalde, M. Noy: Consecutive subwords in permutations, Advances in Applied Mathematics 30 (2003), 110-125.

[29] P. Flajolet: Combinatorial aspects of continued fractions, Disc. Math. 41 (1982), 125-161.

[30] D. Foata and A. Randrianarivony: Two oiseau decompositions of permutations and their application to Eulerian calculus, Europ. J. Combin. 27 (2006), 342-363.

[31] D. Foata and D. Zeilberger: Denert's permutation statistic is indeed Euler-Mahonian, Studies in Appl. Math. 83 (1990),31-59.

[32] D. Foata and D. Zeilberger: Babson-Steingrímsson statistics are indeed Mahonian (and sometimes even Euler-Mahonian), Adv. Applied Math. 27 (2001), no. 2-3, 390-404.

[33] J. Françon and X. G. Viennot: Permutations selon les pics, creux, doubles montées, doubles descentes, nombres d'Euler, nombres de Genocchi, Disc. Math. 28 (1979), 21-35.

[34] I. Gessel:, Symmetric functions and P-recursiveness. J. Combin. Theory Ser. A 53 (1990), $257-285$.

[35] S. Gire: Arbres, permutations à motifs exclus et cartes planaires: quelques problèmes algorithmique et combinatoire, Ph.D. thesis, Université Bordeaux I (1993).

[36] I. Goulden, D. Jackson: Combinatorial Enumeration, Wiley, 1983.

[37] J. Haglund: $q$-rook polynomials and matrices over finite fields, Adv. in Appl. Math. 20 (1998), no. 4, 450-487.

[38] M.T. Hardarson: Avoidance of partially ordered generalized patterns of the form $k-\sigma-k$, arXiv:0805.1872v1

[39] M.T. Hardarson: Personal communication, 2008.

[40] B. Jacquard and G. Schaeffer: A bijective census of nonseparable planar maps, J. Combin. Theory Ser. A, 83, No. 1 (1998), 1-20.

[41] S. Kitaev: Multi-avoidance of generalized patterns, Disc. Math. 260 (2003), 89-100. 
[42] S. Kitaev: Generalized Pattern avoidance with additional restrictions, Sém. Lothar. Combin., B48e (2003), 19 pp.

[43] S. Kitaev and T. Mansour: Partially ordered generalized patterns and $k$-ary words, Ann. Combin. 7 (2003) 191-200,

[44] S. Kitaev: Introduction to partially ordered patterns, Discrete Applied Math. 155 (2007), 929-944.

[45] S. Kitaev: Partially ordered generalized patterns, Disc. Math. 298 (2005), 212-229.

[46] S. Kitaev and T. Mansour: On multi-avoidance of generalized patterns, Ars Combin., 76 (2005), 321-350.

[47] S. Kitaev and T. Mansour: Simultaneous avoidance of generalized patterns, Ars Combin., 75 (2005), 267-288.

[48] P.A. MacMahon: Combinatory Analysis, vols. 1 and 2, Cambridge Univ. Press, Cambridge, 1915 (reprinted by Chelsea,New York, 1955).

[49] A. Marcus and G. Tardos: Excluded permutation matrices and the Stanley-Wilf conjecture, J. Combin. Theory Ser. A 107 (2004), no. 1, 153-160.

[50] A. Mendes: Building generating functions brick by brick, $\mathrm{PhD}$ thesis, University of California, San Diego, (2004).

[51] A. Mendes, J. Remmel: Permutations and words counted by consecutive patterns, Advances in Appl. Math. 37 (2006) 443-480.

[52] J. Noonan, D. Zeilberger: The enumeration of permutations with a prescribed number of "forbidden" patterns, Adv. in Appl. Math. 17 (1996), no. 4, 381-407.

[53] E. Ouchterlony: On Young tableaux involutions and patterns in permutations, Thesis, Matematiska institutionen Linköpings universitet, Linköping, Sweden, 2005.

[54] R. Parviainen: Lattice path enumeration of permutations with $\mathrm{k}$ occurrences of the pattern 2-13, J. Integer Sequences 9 (2006), Article 06.3.2.

[55] A. Regev and Y. Roichman: Generalized statistics on $S_{n}$ and pattern avoidance, European J. Combin. 26 (2005), 29-57.

[56] R. Simion, F. Schmidt: Restricted permutations, European J. Combin. 6 (1985), no. 4, 383406.

[57] R. Simion and D. Stanton: Octabasic Laguerre polynomials and permutation statistics, J. Comput. Appl. Math. 68 (1996), 297-329.

[58] E. Steingrímsson and L. Williams: Permutation tableaux and permutation patterns, J. Combin. Theory Ser. A 114 (2007), 211-234.

[59] J. West: Permutations with forbidden subsequences; and, Stack-sortable permutations, PhDthesis, Massachusetts Institute of Technology (1990).

[60] H. S. Wilf: The patterns of permutations, Disc. Math. 257 (2002) 575-583.

[61] L. Williams: Enumeration of totally positive Grassmann cells, Advances in Math., 190 (2005), 319-342.

[62] A. Woo and A. Yong: When is a Schubert variety Gorenstein?, Adv. Math. 207 (2006), no. $1,205-220$.

[63] D. Zeilberger: The umbral transfer matrix method. I. Foundations, J. Combin. Theory Ser. A 91 (2000), 451-463. 